\title{
0 Poliedro de Euler e as Fases de Gibbs
}

LIONELLO POGLIANI*

\begin{abstract}
Ou melhor, as faces de Euler $e$ as fases de Gibbs. Mostra-se como uma escolha apropriada dos termos permite obter 0 poliedro de Euler a partir das fases e dos componentes de Gibbs

(e vice-versa). Mostra-se também que os poliedros estão para os diagramas de fase como um cubo está para o seu equivalente bidimensional e como é possivel obter o número ciclomático com a regra de Euler.
\end{abstract}

\section{INTRODUÇ̃̃̃O}

J. W. Gibbs (1839-1903) definiu a fase de um sistema químico-físico simples (isto é, um sistema sem campos magnéticos, eléctricos ou gravitacionais) como um estado da matéria uniforme seja na sua composição química seja no seu estado fisico [1] e definiu também o número de componentes $\mathrm{C}$ do sistema como o número mínimo de espécies independentes necessárias para definir a composição de todas as fases $\mathrm{F}$ presentes no sistema e demonstrou de maneira muito elegante que o numero de variáveis independentes $V$ de um sistema de $F$ fases e $\mathrm{C}$ componentes delimitados pelas duas variáveis (temperatura e pressão) que definem o potencial químico de cada componente das $\mathrm{F}$ fases é dado pela famosa regra das fases:

$V=C-F+2$

\section{MÉTODO}

Se definirmos, de maneira um pouco unilateral, a face $\mathrm{F}$ de um sistema poliédrico simples, como o poliedro da figura 1 (um poliedro simples é um poliedro sem buracos de maneira que a sua superficie possa ser transformada por uma série de deformações contínuas na superfície de uma esfera) como um estado geométrico uniforme bidimensional e o número das conexões $\mathrm{C}$ desse mesmo sistema como o numero mínimo de to de todas as faces, e se além disso definirmos os vértices $V$ do sistema poliédrico simples como o número de variáveis desse mesmo sistema constituido por $\mathrm{F}$ faces e por $\mathrm{C}$ conexões delimitadas pelos dois vértices que definem cada um dos lados das F faces, então por uma simples questão de paralelismo formal para que esse estranho 'isomorfismo' (verdadeiro isomorfismo seria a exacta correspondência entre os dois grupos \{variáveis, fases, componentes\} e \{vértices, faces, conexões\} e a possibilidade de resolver os problemas num grupo utilizando os correspondente elementos isomorfos do outro grupo) seja perfeito deve valer também para os poliedros simples a seguinte regra, a que chamaremos regra dos poliedros:

$V=C-F+2$

\section{RESULTADOS}

Se a regra 1 nos diz que o número de fases de um sistema fisico-químico simples nunca poderá superar o número dos componentes mais as duas variáveis que os definem, também a regra 2 nos diz que o número das faces de um poliedro simples nunca poderá superar 0 número de conexões mais os dois vértices que as definem.

Ora, como este estranho "isomorfismo" foi artificialmente construido através de uma escolha apropriada dos termos, ocorre-nos a suspeita de que a regra dos poliedros possa ser falsa e que a sua validade no caso da Figura 1 seja simplesmente uma estranha coincidência devido à forma peculiar do poliedro escolhido e nada mais. Na realidade, a regra é não somente verdadeira como a sua validade foi demonstrada pelo matemático suiço L. Euler (1707-1783) um século antes que J.W. Gibbs obtivesse a sua regra das fases. Experimente-se, com o poliedro da Figura 1, introduzir uma nova conexão entre dois vértices ou eliminar uma já existente: a regra continua a ser obedecida (um método semelhante foi utilizado por Euler para a demonstrar). Essa estranha concordância formal das duas regras (conhecia Gibbs essa regra? Suspeitava da sua utilidade formal para resolver o problema das fases?) tem intrigado muitos cientistas; os dois grupos de V, C e F, porém, não são isomorfos e todas as tentativas para encontrar uma relação entre as duas regras falharam até hoje.

E tudo poderia acabar aqui, com

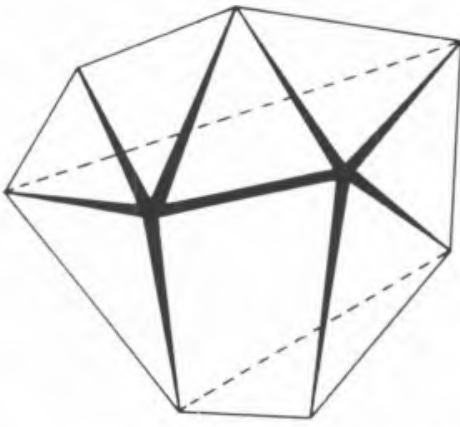

Figura 1: um poliedro simples para 0 qual se verifica a regra dos poliedros: $V=18-11+2=9$.

essa estranheza 'isomórfica', mas na verdade alguns químicos utilizando a teoria dos grafos[2] chegaram a alguns resultados químicos interessantes partindo da formula de Euler dos poliedros simples, ou melhor, partindo dos grafos planares. A teoria dos grafos é uma teoria baseada em elementos de topologia, sendo esta um ramo da geometria que estuda as propriedades das figuras geométricas que se mantém quando as mesmas figuras são submetidas a deformações tão grandes que façam alterar todas as propriedades métricas e projectivas (a topologia é vulgarmente conhecida como a geometria das figuras de borracha); o pai desse tipo de geometria foi 0 astrónomo e matemático alemão A. F. Moebius (1790-1868), que os químicos quânticos bem conhecem devido a um objecto geométrico, 0 anel de Moebius, que acabou por se transformar também num objecto de cálculos de orbitais moleculares [2,3]. Vejamos antes de mais o que é um grafo planar. Um poliedro simples pode ser transformado num certo grafo planar simplesmente achatando-o num plano de maneira a preservar todas as conexões e todos os vértices (deformação permitida em topologia) como acontece com o cubo da Figura 2.

Figura 2: cubo achatado bidimensionalmente para dar o correspondente grafo planar
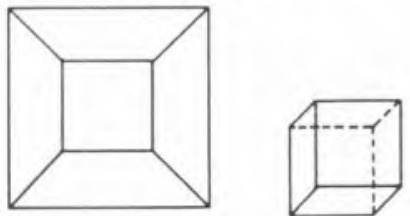
Os grafos planares, descobertos por Euler, ao perderem uma face (imagine-se um cubo de borracha oco; se cortarmos uma face desse poliedro vazio poderemos deformar a sua superficie até deitá-lo completamente sobre um plano) verificam a seguinte regra:

$V=C-F+1$

$\mathrm{Na}$ verdade, topologicamente falando, a regra (2) continua a valer se a face que desapareceu é uma face levada ao infinito, isto é, uma face que se transformou no espaço externo do grafo planar. Se considerarmos, porém, somente 0 reticulado do grafo planar a regra (3) é de utilidade mais directa, como veremos em seguida.

0 diagrama de fases da Figura $3 a$, por exemplo, pode ser visto como um grafo planar G, e como tal pode ser transformado no correspondente grafo dual $\mathrm{G}^{*}$, isto é: se cada região do diagrama de fases, incluindo o seu exterior, for representada por um vértice $V^{*}$, e se duas regiões do diagrama de fases possuem uma conexão $\mathrm{C}$ em comum, então os correspondentes vértices $V^{*}$ podem ser ligados por uma conexão $C^{*}$ (Figura 3b) e 0 grafo assim resultante, Figura $3 c$, é 0 grafo dual do diagrama de fases, obedecendo esse grafo dual à regra (3), pois:

$C^{*}=10, F^{*}=6$ e V* $=5$

Representados como grafos duais, os diagramas das fases podem ser facilmente memorizados e tratados em computador. Deixa-se ao leitor como exercicio a obtenção do grafo dual do diagrama de fases da Figura 4.

A fórmula (3) tem porém uma utilidade ainda mais ampla em Química, especialmente em Química Orgânica. Considerando uma estrutura química sem hidrogénios (hydrogen suppressed graph) como um grafo planar (as fórmulas estruturais que estamos habituados a desenhar para moleculas orgânicas) e pondo: $R=F(R=$ rings), número de anéis; $A=V$, número de átomos, e $B=C$ ( $B=$ bonds), número de ligações, obtemos, por simples rearranjo da regra (3), o chamado o número ciclomático (cyclomatic number) $R$ ou seja, o numero de anéis:

$R=B-(A-1)$

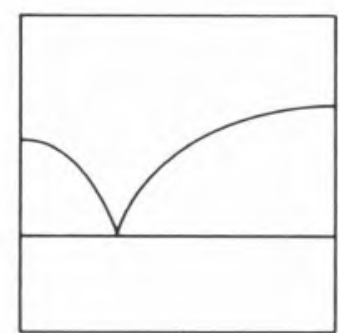

a

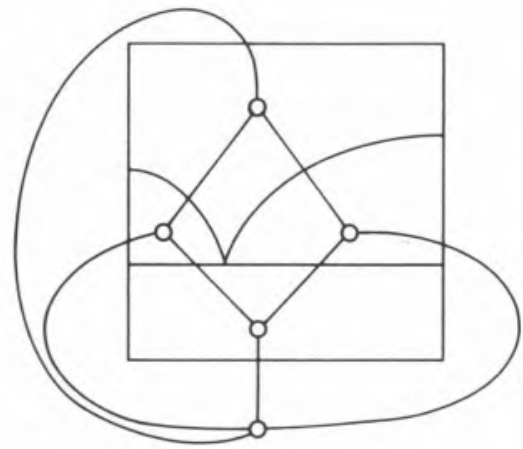

b

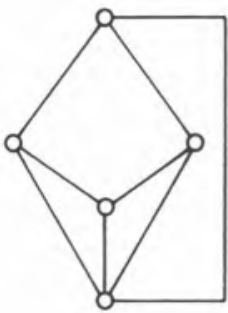

Para um sistema acíclico $B=A-1$ e, claramente, $R=0$,enquanto que em sistemas condensados conhecendo $B$ e A podemos determinar R. A regra (4) tem sido a base para demonstrar a validade geral de uma série de regras que são utilizadas no processo de 'inverse imaging' [4] para reconstruir estruturas químicas a partir das suas propriedades gráficas [5-7]. Por exemplo, a seguinte regra

$B \max =A-[n(M e)-1]$

dá-nos 0 número máximo de ligações, Bmax ,existentes no caminho mais longo possivel de um grafo molecular cujo número de átomos de carbono é $\mathrm{A}$ e cujo número de grupos metílicos é $\mathrm{n}(\mathrm{Me})$.

$$
\text { Exemplo: } \mathrm{C}_{5}(\mathrm{Me})_{7}, \mathrm{Bmax}=6 \text {. }
$$

\section{CONCLUSÃO}

Se a regra das fases de Gibbs não tem utilidade em geometria, pois a geometria nada tem a ver com fases, componentes químicos e variáveis termodinâmicas, já a regra dos poliedros de Euler, como acabamos de ver, é útil em Química, pois estruturas químicas muito diversas, como um diagrama de fases $e$

Figura 4: determine-se 0 grafo dual do seguinte diagrama das fases, visto como um grafo planar.

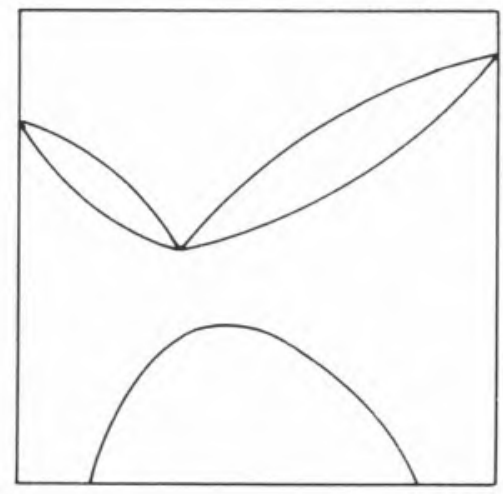

Figura 3: (a) um diagrama de fases, (b) o diagrama de fases com 0 grafo dual sobreposto, (c) 0 grafo dual do diagrama de fases.

uma fórmula molecular, podem ser igualmente vistas como conjuntos de vértices, conexões e faces.

Para concluir este artigo sobre uma tão curiosa mas útil coincidência entre geometria e química, nada melhor do que mencionar uma outra aproveitável coincidência entre letras do alfabeto, números e propinas:sim, e como é lindo finalizar um pesado curso que custa propinas terriveis remidas estudando $=$ 3,14159265358979 que são os primeiros 15 algarismos do número $\pi$ (transforme-se cada palavra no correspondente número de letras) que pode ser utilizado para produzir números aleatórios, tendo esse primeiros 15 números uma média de 5,1 e um desvio padrão de 2,8.

\section{BIBLIOGRAFIA}

1. P. W. Atkins, Physical Chemistry, 3rd ed. Oxford University Press, Oxford, 1987.

2. N. Trinajstic, Chemical Graph Theory, CRC Press, Bocca Raton FL, 1983.

3. M. J. S. Dewar, R. C. Dougherty, The PMO Theory of Organic Chemistry, Plenum Press, New York, 1979.

4. L. B. Kier, L. H. Hall, J. W. Frazer, J. Chem. Int Comp. Chem. , 33 (1993) 143, 148.

5. N. J. Turro, Angew. Chem. Int. Ed. Engl. 25 (1986) 882.

6. P. J. Hansen, P. J. C. Jurs, J.Chem. Educ, 65 (1988) 574 .

7. L. Pogliani, Chemistry and Properties of Biomolecular Systems, N. Russo et al.(eds), Vol. 2, Kluwer, Dordrecht, 1993.

*Centro de Química-Física Molecular, Instituto Superior Técnico, 1096 Lisboa Codex.

Em gozo de licença sabática do Dipartimento di Chimica, Università della Calabria, 87030 Rende (CS), Itália. 